\title{
ON A THIRD ORDER DIFFERENCE EQUATION
}

\author{
R. ABo-ZEID
}

Abstract. In this paper, we solve the difference equation

$$
x_{n+1}=\frac{x_{n} x_{n-2}}{-a x_{n}+b x_{n-2}}, \quad n=0,1, \ldots
$$

where $a$ and $b$ are positive real numbers and the initial values $x_{-2}, x_{-1}$ and $x_{0}$ are real numbers. We find invariant sets and discuss the global behavior of the solutions of that equation. We show that when $a>\frac{4}{27} b^{3}$, under certain conditions there exist solutions, either periodic or converge to periodic solutions.

2010 Mathematics Subject Classification: 39A20, 39A21.

Keywords: difference equation, forbidden set, convergence,bounded, unbounded solution.

\section{INTRODUCTION}

In their paper [9], the authors studied some special cases of the difference equation

$$
x_{n+1}=\frac{\alpha+\beta x_{n} x_{n-1}+\gamma x_{n-1}}{A+B x_{n} x_{n-1}+C x_{n-1}}, \quad n=0,1, \ldots,
$$

with nonnegative parameters and with arbitrary nonnegative initial conditions such that the denominator is always positive. In [15], Dehghan, et al. studied the global attractivity of the positive equilibrium of some special cases that contains at least one quaderatic term of the second order rational difference equations

$$
x_{n+1}=\frac{A x_{n}^{2}+B x_{n} x_{n-1}+C x_{n-1}^{2}+D x_{n}+E x_{n-1}+F}{\alpha x_{n}+\beta x_{n-1}+\gamma}, \quad n=0,1, \ldots,
$$

which has quadratic terms in their numerators and linear terms in their denominators. In [17], the authors investigated the global behaviour of non-negative solutions 
of the rational difference equation with arbitrary delay and quadratic terms in its numerator:

$$
x_{n+1}=\frac{A x_{n}^{2}+B x_{n} x_{n-k}+C x_{n-k}^{2}+D x_{n}+E x_{n-k}}{\alpha x_{n}+\beta x_{n-k}+\gamma}, \quad n=0,1, \ldots,
$$

with $k \in\{1,2, \ldots\}$, where all parameters are non-negative, with $A+B+C+D+E>0$ and $\gamma>0$.

In [2], we have studied the behavior of the solutions of the difference equation

$$
x_{n+1}=\frac{a x_{n} x_{n-1}}{-b x_{n}+c x_{n-2}}, \quad n=0,1, \ldots,
$$

where $a, b, c$ are positive real numbers and the initial conditions $x_{0}, x_{-1}, x_{-2}$ are real numbers. Also, in [6] we have studied the global behavior of the fourth order difference equation

$$
x_{n+1}=\frac{a x_{n} x_{n-2}}{-b x_{n}+c x_{n-3}}, \quad n=0,1, \ldots,
$$

where $a, b, c$ are positive real numbers and the initial conditions $x_{0}, x_{-1}, x_{-2}, x_{-3}$ are real numbers. For more publications on global behavior of the solutions and forbidden sets, one can see [1]- [23].

In this paper, we shall determine the forbidden set, find the solution and investigate the behavior of the solutions of the equation

$$
x_{n+1}=\frac{x_{n} x_{n-2}}{-a x_{n}+b x_{n-2}}, \quad n=0,1, \ldots,
$$

where $a$ and $b$ are positive real numbers and the initial values $x_{-2}, x_{-1}$ and $x_{0}$ are real numbers.

\section{Solution of EQUATion (1)}

The reciprocal transformation

$$
x_{n}=\frac{1}{y_{n}}
$$

reduces equation (1) into the third order linear homogeneous difference equation

$$
y_{n+1}-b y_{n}+a y_{n-2}=0, \quad n=0,1 \ldots
$$

The characteristic equation of equation (2) is

$$
\lambda^{3}-b \lambda^{2}+a=0 .
$$


Clear that equation (3) has a negative real root $\lambda_{0}$ for all values of $(a, b>0)$. Therefore, the roots of equation (3) are

$$
\lambda_{0}, \quad \lambda_{ \pm}=-\frac{\lambda_{0}-b}{2} \pm \frac{\sqrt{\left(\lambda_{0}-b\right)^{2}-4 \lambda_{0}\left(\lambda_{0}-b\right)}}{2} .
$$

The roots of equation (3) depends on the relation between $a$ and $b$.

Lemma 1. For equation (3), we have the following:

1. If $a>\frac{4}{27} b^{3}$, then equation (3) has one negative real root and two complex conjugate roots.

2. If $a=\frac{4}{27} b^{3}$, then equation (3) has one negative real root and a repeated positive real root.

3. If $a<\frac{4}{27} b^{3}$, then equation (3) has three real different roots, one of them is negative and two positive roots.

Proof. It is sufficient to see that, the discriminant of the polynomial

$$
p(\lambda)=\lambda^{3}-b \lambda^{2}+a=0
$$

is

$$
\triangle=-4 b^{3} a+27 a^{2} .
$$

We shall consider the three cases given in lemma (1).

Case $a>\frac{4}{27} b^{3}$ :

When $a>\frac{4}{27} b^{3}$, the roots of equation (3) are

$$
\lambda_{0}<-\frac{b}{3}, \quad \lambda_{ \pm}=-\frac{\lambda_{0}-b}{2} \pm i \frac{\sqrt{4 \lambda_{0}\left(\lambda_{0}-b\right)-\left(\lambda_{0}-b\right)^{2}}}{2} .
$$

Then the solution of equation (1) is

$$
x_{n}=\frac{1}{c_{1} \lambda_{0}^{n}+\left(\frac{-a}{\lambda_{0}}\right)^{\frac{n}{2}}\left(c_{2} \cos n \varphi+c_{3} \sin n \varphi\right)},
$$

where

$$
\left.\left|\lambda_{ \pm}\right|=\sqrt{\lambda_{0}\left(\lambda_{0}-b\right)}=\sqrt{\frac{-a}{\lambda_{0}}} \text { and } \varphi=\tan ^{-1}\left(\sqrt{\frac{3 \lambda_{0}+b}{\lambda_{0}-b}}\right) \in\right] 0, \frac{\pi}{2}[.
$$


Using the initials $x_{-2}, x_{-1}$ and $x_{0}$, the values of $c_{1}, c_{2}$ and $c_{3}$ are:

$$
\begin{aligned}
& c_{1}=\frac{1}{\Delta_{1}}\left(c_{11} \frac{1}{x_{0}}+c_{12} \frac{1}{x_{-1}}+c_{13} \frac{1}{x_{-2}}\right), \\
& c_{2}=\frac{1}{\Delta_{1}}\left(c_{21} \frac{1}{x_{0}}+c_{22} \frac{1}{x_{-1}}+c_{23} \frac{1}{x_{-2}}\right) \\
& \text { and } \\
& c_{3}=\frac{1}{\Delta_{1}}\left(c_{31} \frac{1}{x_{0}}+c_{32} \frac{1}{x_{-1}}+c_{33} \frac{1}{x_{-2}}\right),
\end{aligned}
$$

where

$$
\begin{aligned}
& c_{11}=\frac{\lambda_{0}}{a} \sqrt{-\frac{\lambda_{0}}{a}} \sin \varphi, \quad c_{12}=-\frac{\lambda_{0}}{a} \sin 2 \varphi, \quad c_{13}=-\sqrt{-\frac{\lambda_{0}}{a}} \sin \varphi, \\
& c_{21}=-\frac{1}{a} \sin 2 \varphi-\frac{1}{\lambda_{0}^{2}} \sqrt{-\frac{\lambda_{0}}{a}} \sin \varphi, \quad c_{22}=\frac{\lambda_{0}}{a} \sin 2 \varphi, \quad c_{23}=\sqrt{-\frac{\lambda_{0}}{a}} \sin \varphi, \\
& c_{31}=-\frac{1}{a} \cos 2 \varphi-\frac{1}{\lambda_{0}^{2}} \sqrt{-\frac{\lambda_{0}}{a}} \cos \varphi, \quad c_{32}=\frac{\lambda_{0}}{a} \cos 2 \varphi+\frac{1}{\lambda_{0}^{2}}, \quad c_{33}=\sqrt{-\frac{\lambda_{0}}{a}} \cos \varphi-\frac{1}{\lambda_{0}}
\end{aligned}
$$

and

$$
\Delta_{1}=\left|\begin{array}{ccc}
1 & 1 & 0 \\
\frac{1}{\lambda_{0}} & \sqrt{-\frac{\lambda_{0}}{a}} \cos \varphi & -\sqrt{-\frac{\lambda_{0}}{a}} \sin \varphi \\
\frac{1}{\lambda_{0}^{2}} & -\frac{\lambda_{0}}{a} \cos 2 \varphi & \frac{\lambda_{0}}{a} \sin 2 \varphi
\end{array}\right| .
$$

By simple calculations, we can write the solution of equation (1) as

$$
x_{n}=\frac{1}{\frac{\alpha_{1 n}}{x_{0}}+\frac{\alpha_{2 n}}{x_{-1}}+\frac{\alpha_{3 n}}{x_{-2}}},
$$

where

$$
\begin{aligned}
& \alpha_{1 n}=\frac{1}{\Delta_{1}}\left(c_{11} \lambda_{0}^{n}+c_{21}\left(\frac{-a}{\lambda_{0}}\right)^{\frac{n}{2}} \cos n \varphi+c_{31}\left(\frac{-a}{\lambda_{0}}\right)^{\frac{n}{2}} \sin n \varphi\right), \\
& \alpha_{2 n}=\frac{1}{\Delta_{1}}\left(c_{12} \lambda_{0}^{n}+c_{22}\left(\frac{-a}{\lambda_{0}}\right)^{\frac{n}{2}} \cos n \varphi+c_{32}\left(\frac{-a}{\lambda_{0}}\right)^{\frac{n}{2}} \sin n \varphi\right) \\
& \text { and } \\
& \alpha_{3 n}=\frac{1}{\Delta_{1}}\left(c_{13} \lambda_{0}^{n}+c_{23}\left(\frac{-a}{\lambda_{0}}\right)^{\frac{n}{2}} \cos n \varphi+c_{33}\left(\frac{-a}{\lambda_{0}}\right)^{\frac{n}{2}} \sin n \varphi\right)
\end{aligned}
$$

are such that $c_{i j}, i, j=1,2,3$ are given in (6).

Case $a=\frac{4}{27} b^{3}$ :

When $a=\frac{4}{27} b^{3}$, equation (3) has a negative root $\lambda_{0}=-\frac{b}{3}$ and a repeated positive $\operatorname{root} \frac{2 b}{3}$.

Then the solution of equation (1) is

$$
x_{n}=\frac{1}{c_{1}\left(-\frac{b}{3}\right)^{n}+c_{2}\left(\frac{2 b}{3}\right)^{n}+c_{3}\left(\frac{2 b}{3}\right)^{n} n} .
$$


Using the initials $x_{-2}, x_{-1}$ and $x_{0}$, the values of $c_{1}, c_{2}$ and $c_{3}$ in this case are:

$$
\begin{aligned}
& c_{1}=\frac{1}{\Delta_{2}}\left(c_{11} \frac{1}{x_{0}}+c_{12} \frac{1}{x_{-1}}+c_{13} \frac{1}{x_{-2}}\right), \\
& c_{2}=\frac{1}{\Delta_{2}}\left(c_{21} \frac{1}{x_{0}}+c_{22} \frac{1}{x_{-1}}+c_{23} \frac{1}{x_{-2}}\right) \\
& \text { and } \\
& c_{3}=\frac{1}{\Delta_{2}}\left(c_{31} \frac{1}{x_{0}}+c_{32} \frac{1}{x_{-1}}+c_{33} \frac{1}{x_{-2}}\right),
\end{aligned}
$$

where

$$
\begin{array}{lll}
c_{11}=-\frac{27}{8 b^{3}}, & c_{12}=\frac{9}{2 b^{2}}, & c_{13}=-\frac{3}{2 b} \\
c_{21}=-\frac{27}{b^{3}}, & c_{22}=-\frac{9}{2 b^{2}}, & c_{23}=\frac{3}{2 b} \\
c_{31}=-\frac{81}{4 b^{3}}, & c_{32}=\frac{27}{4 b^{2}}, & c_{33}=\frac{9}{2 b}
\end{array}
$$

and

$$
\Delta_{2}=\left|\begin{array}{ccc}
1 & 1 & 0 \\
\left(-\frac{3}{b}\right) & \left(\frac{3}{2 b}\right) & -\left(\frac{3}{2 b}\right) \\
\left(-\frac{3}{b}\right)^{2} & \left(\frac{3}{2 b}\right)^{2} & -2\left(\frac{3}{2 b}\right)^{2}
\end{array}\right| .
$$

By simple calculations, we can write the solution of equation (1) in this case as

$$
x_{n}=\frac{1}{\frac{\alpha_{1 n}}{x_{0}}+\frac{\alpha_{2 n}}{x_{-1}}+\frac{\alpha_{3 n}}{x_{-2}}}
$$

where

$$
\begin{aligned}
& \alpha_{1 n}=\frac{1}{\Delta_{2}}\left(c_{11}\left(-\frac{b}{3}\right)^{n}+c_{21}\left(\frac{2 b}{3}\right)^{n}+c_{31}\left(\frac{2 b}{3}\right)^{n} n,\right. \\
& \alpha_{2 n}=\frac{1}{\Delta_{2}}\left(c_{12}\left(-\frac{b}{3}\right)^{n}+c_{22}\left(\frac{2 b}{3}\right)^{n}+c_{32}\left(\frac{2 b}{3}\right)^{n} n\right. \\
& \text { and } \\
& \alpha_{3 n}=\frac{1}{\Delta_{2}}\left(c_{13}\left(-\frac{b}{3}\right)^{n}+c_{23}\left(\frac{2 b}{3}\right)^{n}+c_{33}\left(\frac{2 b}{3}\right)^{n} n\right.
\end{aligned}
$$

are such that $c_{i j}, i, j=1,2,3$ are given in (12).

Case $a<\frac{4}{27} b^{3}$ :

When $a<\frac{4}{27} b^{3}$, the roots of equation (3) are

$$
\lambda_{0}>-\frac{b}{3}, \quad \lambda_{ \pm}=-\frac{\lambda_{0}-b}{2} \pm \frac{\sqrt{\left(\lambda_{0}-b\right)^{2}-4 \lambda_{0}\left(\lambda_{0}-b\right)}}{2},
$$

where

$$
\lambda_{+}>\lambda_{-}>\left|\lambda_{0}\right|>0
$$

Then the solution of equation (1) is 


$$
x_{n}=\frac{1}{c_{1} \lambda_{0}^{n}+c_{2} \lambda_{-}^{n}+c_{3} \lambda_{+}^{n}} .
$$

Using the initials $x_{-2}, x_{-1}$ and $x_{0}$, the values of $c_{1}, c_{2}$ and $c_{3}$ in this case are:

$$
\begin{aligned}
& c_{1}=\frac{1}{\Delta_{3}}\left(c_{11} \frac{1}{x_{0}}+c_{12} \frac{1}{x_{-1}}+c_{13} \frac{1}{x_{-2}}\right), \\
& c_{2}=\frac{1}{\Delta_{3}}\left(c_{21} \frac{1}{x_{0}}+c_{22} \frac{1}{x_{-1}}+c_{23} \frac{1}{x_{-2}}\right) \\
& \text { and } \\
& c_{3}=\frac{1}{\Delta_{3}}\left(c_{31} \frac{1}{x_{0}}+c_{32} \frac{1}{x_{-1}}+c_{33} \frac{1}{x_{-2}}\right),
\end{aligned}
$$

where

$$
\begin{aligned}
& c_{11}=\frac{\lambda_{-}-\lambda_{+}}{\lambda_{-}^{2} \lambda_{+}^{2}}, \quad c_{12}=\frac{-\lambda_{-}^{2}+\lambda_{+}^{2}}{\lambda_{-}^{2} \lambda_{+}^{2}}, \quad c_{13}=\frac{\lambda_{-}-\lambda_{+}}{\lambda_{-} \lambda_{+}}, \\
& c_{21}=\frac{\lambda_{+}-\lambda_{0}}{\lambda_{+}^{2} \lambda_{0}^{2}}, \quad c_{22}=\frac{\lambda_{0}^{2}-\lambda_{+}^{2}}{\lambda_{+}^{2} \lambda_{0}^{2}}, \quad c_{23}=\frac{\lambda_{+}-\lambda_{0}}{\lambda_{+} \lambda_{0}}, \\
& c_{31}=\frac{\lambda_{0}-\lambda_{-}}{\lambda_{0}^{2} \lambda_{-}^{2}}, \quad c_{32}=\frac{\lambda_{-}^{2}-\lambda_{0}^{2}}{\lambda_{0}^{2} \lambda_{-}^{2}}, \quad c_{33}=\frac{\lambda_{0}-\lambda_{-}}{\lambda_{0} \lambda_{-}}
\end{aligned}
$$

and

$$
\Delta_{3}=\left|\begin{array}{ccc}
1 & 1 & 1 \\
\frac{1}{\lambda_{0}} & \frac{1}{\lambda_{-}} & \frac{1}{\lambda_{+}} \\
\frac{1}{\lambda_{0}^{2}} & \frac{1}{\lambda_{-}^{2}} & \frac{1}{\lambda_{+}^{2}}
\end{array}\right|
$$

By simple calculations, we can write the solution of equation (1) in this case as

$$
x_{n}=\frac{1}{\frac{\alpha_{1 n}}{x_{0}}+\frac{\alpha_{2 n}}{x_{-1}}+\frac{\alpha_{3 n}}{x_{-2}}},
$$

where

$$
\begin{aligned}
& \alpha_{1 n}=\frac{1}{\Delta_{3}}\left(c_{11} \lambda_{0}^{n}+c_{21} \lambda_{-}^{n}+c_{31} \lambda_{+}^{n}\right), \\
& \alpha_{2 n}=\frac{1}{\Delta_{3}}\left(c_{12} \lambda_{0}^{n}+c_{22} \lambda_{-}^{n}+c_{32} \lambda_{+}^{n}\right) \\
& \text { and } \\
& \alpha_{3 n}=\frac{1}{\Delta_{3}}\left(c_{13} \lambda_{0}^{n}+c_{23} \lambda_{-}^{n}+c_{33} \lambda_{+}^{n}\right)
\end{aligned}
$$

are such that $c_{i j}, i, j=1,2,3$ are given in (17).

Using equations (8), (13) and (18), we can write the forbidden set of equation (1) as

$$
F=\bigcup_{n=-2}^{\infty}\left\{\left(x_{0}, x_{-1}, x_{-2}\right) \in \mathbb{R}^{3}: \frac{\alpha_{1 n}}{x_{0}}+\frac{\alpha_{2 n}}{x_{-1}}+\frac{\alpha_{3 n}}{x_{-2}}=0\right\},
$$


where $\alpha_{1 n}, \alpha_{2 n}$ and $\alpha_{3 n}$ are given as follows:

$$
\begin{cases}\alpha_{1 n}, \alpha_{2 n} \text { and } \alpha_{3 n} \text { are given in (9), } & a>\frac{4}{27} b^{3} \\ \alpha_{1 n}, \alpha_{2 n} \text { and } \alpha_{3 n} \text { are given in (14), } & a=\frac{4}{27} b^{3} \\ \alpha_{1 n}, \alpha_{2 n} \text { and } \alpha_{3 n} \text { are given in (19), } & a<\frac{4}{27} b^{3} .\end{cases}
$$

\section{Global Behavior of equation (1)}

Consider the set

$$
D=\left\{(x, y, z) \in \mathbb{R}^{3}: \frac{\lambda^{2}}{x}-\frac{a}{y}-\frac{a \lambda}{z}=0\right\}
$$

with

$$
\begin{cases}\lambda=\lambda_{0}, & a>\frac{4}{27} b^{3} \\ \lambda=-\frac{b}{3}, & a=\frac{4}{27} b^{3} .\end{cases}
$$

Clear that, when $a=\frac{4}{27} b^{3}$, the set $D$ can be written as

$$
D=\left\{(x, y, z) \in \mathbb{R}^{3}: \frac{9}{x}-\frac{12 b}{y}+\frac{4 b^{2}}{z}=0\right\}
$$

Note that, for the point $(x, y, z) \in \mathbb{R}^{3}$, the relation $\frac{\lambda_{0}^{2}}{x}+\frac{a}{y}+\frac{a \lambda_{0}}{z}=0$ is equivalent to $c_{1}(x, y, z)=0$, where $c_{1}$ is given by either (5) or (11) according to the relations $a>\frac{4}{27} b^{3}$ and $a=\frac{4}{27} b^{3}$ respectively.

Theorem 2. The set $D$ is an invariant for equation (1).

Proof. Let $\left(x_{0}, x_{-1}, x_{-2}\right) \in D$. We show that $\left(x_{k}, x_{k-1}, x_{k-2}\right) \in D$ for each $k \in N$. The proof is by induction on $k$. The point $\left(x_{0}, x_{-1}, x_{-2}\right) \in D$, implies

$$
\frac{\lambda_{0}^{2}}{x_{0}}-\frac{a}{x_{-1}}-\frac{a \lambda_{0}}{x_{-2}}=0
$$

Now for $k=1$, we have

$$
\begin{aligned}
& \frac{\lambda_{0}^{2}}{x_{1}}-\frac{a}{x_{0}}-\frac{a \lambda_{0}}{x_{-1}}=\frac{\lambda_{0}^{2}}{x_{0} x_{-2}}\left(-a x_{0}+b x_{-2}\right)-\frac{a}{x_{0}}-\frac{a \lambda_{0}}{x_{-1}} \\
= & \frac{1}{x_{0} x_{-1} x_{-2}}\left(-a \lambda_{0}^{2} x_{0} x_{-1}+b \lambda_{0}^{2} x_{-1} x_{-2}-a x_{-1} x_{-2}-a \lambda_{0} x_{0} x_{-2}\right) \\
= & \frac{1}{x_{0} x_{-1} x_{-2}}\left(-a \lambda_{0}^{2} x_{0} x_{-1}+\left(\lambda_{0}^{2} b-a\right) x_{-1} x_{-2}-a \lambda_{0} x_{0} x_{-2}\right)
\end{aligned}
$$




$$
\begin{gathered}
=\frac{1}{x_{0} x_{-1} x_{-2}}\left(-a \lambda_{0}^{2} x_{0} x_{-1}+\lambda_{0}^{3} x_{-1} x_{-2}-a \lambda_{0} x_{0} x_{-2}\right) \\
=\lambda_{0}\left(\frac{\lambda_{0}^{2}}{x_{0}}-\frac{a}{x_{-1}}-\frac{a \lambda_{0}}{x_{-2}}\right)=0 .
\end{gathered}
$$

This implies that $\left(x_{1}, x_{0}, x_{-1}\right) \in D$.

Suppose that the $\left(x_{k}, x_{k-1}, x_{k-2}\right) \in D$. That is

$$
\frac{\lambda_{0}^{2}}{x_{k}}-\frac{a}{x_{k-1}}-\frac{a \lambda_{0}}{x_{k-2}}=0
$$

Then

$$
\begin{gathered}
\frac{\lambda_{0}^{2}}{x_{k+1}}-\frac{a}{x_{k}}-\frac{a \lambda_{0}}{x_{k-1}}=\frac{\lambda_{0}^{2}}{x_{k} x_{k-2}}\left(-a x_{k}+b x_{k-2}\right)-\frac{a}{x_{k}}-\frac{a \lambda_{0}}{x_{k-1}} \\
=\frac{1}{x_{k} x_{k-1} x_{k-2}}\left(-a \lambda_{0}^{2} x_{k} x_{k-1}+b \lambda_{0}^{2} x_{k-1} x_{k-2}-a x_{k-1} x_{k-2}-a \lambda_{0} x_{k} x_{k-2}\right) \\
=\frac{1}{x_{k} x_{k-1} x_{k-2}}\left(-a \lambda_{0}^{2} x_{k} x_{k-1}+\left(\lambda_{0}^{2} b-a\right) x_{k-1} x_{k-2}-a \lambda_{0} x_{k} x_{k-2}\right) \\
=\frac{1}{x_{k} x_{k-1} x_{k-2}}\left(-a \lambda_{0}^{2} x_{k} x_{k-1}+\lambda_{0}^{3} x_{k-1} x_{k-2}-a \lambda_{0} x_{k} x_{k-2}\right) \\
=\lambda_{0}\left(\frac{\lambda_{0}^{2}}{x_{k}}-\frac{a}{x_{k-1}}-\frac{a \lambda_{0}}{x_{k-2}}\right)=0 .
\end{gathered}
$$

Therefore, $\left(x_{k+1}, x_{k}, x_{k-1}\right) \in D$.

This completes the proof.

Now assume that $a<\frac{4}{27} b^{3}$. We shall consider the three sets

$$
D_{i}=\left\{(x, y, z) \in \mathbb{R}^{3}: \frac{\lambda^{2}}{x}-\frac{a}{y}-\frac{a \lambda}{z}=0\right\}, \quad i=1,2,3,
$$

with

$$
\begin{cases}\lambda=\lambda_{0}, & \mathrm{i}=1 \\ \lambda=\lambda_{-}, & \mathrm{i}=2 \\ \lambda=\lambda_{+}, & \mathrm{i}=3 .\end{cases}
$$

By simple calculations, we can see that:

$$
\begin{cases}D_{i} \text { is equivalent to } c_{1}(x, y, z)=0, & \mathrm{i}=1 ; \\ D_{i} \text { is equivalent to } c_{2}(x, y, z)=0, & \mathrm{i}=2 ; \\ D_{i} \text { is equivalent to } c_{1}(x, y, z)=0, & \mathrm{i}=3,\end{cases}
$$

where $c_{i}, i=1,2$ and 3 are given by (16). 
Theorem 3. Each set of the sets $D_{i}, i=1,2$ and 3 is an invariant for equation (1).

Proof. The proof is similar to that of theorem (2) and will be omitted.

Theorem 4. Let $\left\{x_{n}\right\}_{n=-2}^{\infty}$ be a solution of equation (1) such that $\left(x_{0}, x_{-1}, x_{-2}\right) \notin$ $F \cup D$. If $a>\frac{4}{27} b^{3}$, then we have the following:

1. If $a \geq b+1$, then $\left\{x_{n}\right\}_{n=-2}^{\infty}$ converges to zero.

2. If $a<b+1$, then we have the following:

(a) If $a \geq 1$, then $\left\{x_{n}\right\}_{n=-2}^{\infty}$ converges to zero.

(b) If $a<1$, then we have the following:

i. If $a^{2}+a b-1>0$, then $\left\{x_{n}\right\}_{n=-2}^{\infty}$ converges to zero.

ii. If $a^{2}+a b-1=0$, then $\left\{x_{n}\right\}_{n=-2}^{\infty}$ is bounded.

iii. If $a^{2}+a b-1<0$, then $\left\{x_{n}\right\}_{n=-2}^{\infty}$ is unbounded.

Proof. The solution of equation (1) when $a>\frac{4}{27} b^{3}$ is

$$
x_{n}=\frac{1}{\left.c_{1} \lambda_{0}^{n}+\left(-\frac{a}{\lambda_{0}}\right)^{\frac{n}{2}}\left(c_{2} \cos n \varphi+c_{3} \sin n \varphi\right)\right)} .
$$

1. When $a>b+1$, we have $-a<-\sqrt[3]{a}<\lambda_{0}<-1$. That is $\left(\frac{-a}{\lambda_{0}}\right)^{n} \rightarrow \infty$ and $\lambda_{0}^{n}$ is unbounded.

If $a=b+1$, then we have that $-a<-\sqrt[3]{a}<\lambda_{0}=-1$. That is $\left(\frac{-a}{\lambda_{0}}\right)^{n} \rightarrow \infty$ as $n \rightarrow \infty$ and the result follows.

2. When $a<b+1$, we have that $\lambda_{0}>-1$.

(a) If $a \geq 1$, then $-a \leq-\sqrt[3]{a} \leq-1<\lambda_{0}$. That is $\left(\frac{-a}{\lambda_{0}}\right)^{n} \rightarrow \infty$, from which the result follows.

(b) If $a<1$, then $a<\sqrt[3]{a}$ and we have the following:

i. If $a^{2}+a b-1>0$, then $\lambda_{0}>-a>-\sqrt[3]{a}>-1$. This implies that $\lambda_{0}^{n} \rightarrow 0$ and $\left(\frac{-a}{\lambda_{0}}\right)^{n} \rightarrow \infty$, from which the result follows.

ii. If $a^{2}+a b-1=0$, then $\lambda_{0}=-a>-\sqrt[3]{a}>-1$. That is $\lambda_{0}^{n} \rightarrow 0$.

But as

$$
\left|c_{1} \lambda_{0}^{n}+c_{2} \cos n \varphi+c_{3} \sin n \varphi\right| \neq 0 \text { for all } n \geq 0,
$$

the quantity (20) attains its infemum value say $\epsilon>0$ and the result follows. 
iii. If $a^{2}+a b-1<0$, then $-a>\lambda_{0}>-\sqrt[3]{a}>-1$. This implies that $\lambda_{0}^{n} \rightarrow 0$ and $\left(\frac{-a}{\lambda_{0}}\right)^{n} \rightarrow 0$, from which the result follows.

Theorem 5. Let $\left\{x_{n}\right\}_{n=-2}^{\infty}$ be a solution of equation (1) such that $\left(x_{0}, x_{-1}, x_{-2}\right) \notin$ $F \cup D$. If $a=\frac{4}{27} b^{3}$, then we have the following:

1. If $a \geq b+1$, then $\left\{x_{n}\right\}_{n=-2}^{\infty}$ converges to zero.

2. If $a<b+1$, then we have the following:

(a) If $0<b<\frac{3}{2}$, then $\left\{x_{n}\right\}_{n=-2}^{\infty}$ is unbounded.

(b) If $\frac{3}{2} \leq b<3$, then $\left\{x_{n}\right\}_{n=-2}^{\infty}$ converges to zero.

Proof. The solution of equation (1) when $a=\frac{4}{27} b^{3}$ is

$$
x_{n}=\frac{1}{c_{1}\left(-\frac{b}{3}\right)^{n}+c_{2}\left(\frac{2 b}{3}\right)^{n}+c_{3}\left(\frac{2 b}{3}\right)^{n} n} .
$$

1. When $a \geq b+1$, it is sufficient to see that $\lambda_{0}=-\frac{b}{3} \leq-1$ and the result follows.

2. When $a<b+1$, we have that $\lambda_{0}=-\frac{b}{3}>-1$.

(a) If $0<b<\frac{3}{2}$, then $\frac{b}{3}<\frac{1}{2}$ and $\frac{2 b}{3}<1$, from which the result follows.

(b) If $\frac{3}{2} \leq b<3$, then $\frac{1}{2} \leq \frac{b}{3} \leq 1$ and $1 \leq \frac{2 b}{3} \leq 2$, from which the result follows.

Theorem 6. Let $\left\{x_{n}\right\}_{n=-2}^{\infty}$ be a solution of equation (1) such that $\left(x_{0}, x_{-1}, x_{-2}\right) \notin$ $F \cup D_{3}$. If $a<\frac{4}{27} b^{3}$, then we have the following:

1. If $a>-1+b$, then we have the following:

(a) If $0<b<\frac{3}{2}$, then $\left\{x_{n}\right\}_{n=-2}^{\infty}$ is unbounded.

(b) If $b>\frac{3}{2}$, then $\left\{x_{n}\right\}_{n=-2}^{\infty}$ converges to zero.

2. If $a=-1+b$, then we have the following:

(a) If $1 \leq b<\frac{3}{2}$, then $\left\{x_{n}\right\}_{n=-2}^{\infty}$ converges to the $\frac{1}{c_{3}}$.

(b) If $b>\frac{3}{2}$, then $\left\{x_{n}\right\}_{n=-2}^{\infty}$ converges to zero. 
3. If $a<-1+b$, then $\left\{x_{n}\right\}_{n=-2}^{\infty}$ converges to zero.

Proof. Let $f(\lambda)=\lambda^{3}-b \lambda^{2}+a$. It is clear that $f(\lambda)$ is increasing on $]-\infty[, 0 \cup] \frac{2 b}{3}, \infty[$ and decreasing on $] 0, \frac{2 b}{3}$. The solution of equation (1) when $a<\frac{4}{27} b^{3}$ is

$$
x_{n}=\frac{1}{c_{1} \lambda_{0}^{n}+c_{2} \lambda_{-}^{n}+c_{3} \lambda_{+}^{n}} .
$$

We have also

$$
0<\left|\lambda_{0}\right|<\lambda_{-}<\frac{2 b}{3}<\lambda_{+}
$$

The condition $\left(x_{0}, x_{-1}, x_{-2}\right) \notin F \cup D_{3}$ ensures that $c_{3} \neq 0$.

1. When $a>-1+b$, we have two cases:

(a) If $0<b<\frac{3}{2}$, then $\frac{2 b}{3}<\lambda_{+}<1$ (otherwise $a<-1+b$, which is a contradiction). Then $0<\left|\lambda_{0}\right|<\lambda_{-}<\frac{2 b}{3}<\lambda_{+}<1$, from which the result follows.

(b) If $b>\frac{3}{2}$, then $1<\lambda-<\frac{2 b}{3}<\lambda_{+}$and the result follows.

2. If $a=-1+b$, then either $\lambda_{-}=1$ or $\lambda_{+}=1$.

(a) If $1 \leq b<\frac{3}{2}$, then $\lambda_{+}=1$. That is $0<\left|\lambda_{0}\right|<\lambda-<\frac{2 b}{3}<\lambda_{+}=1$. Then

$$
x_{n}=\frac{1}{c_{1} \lambda_{0}^{n}+c_{2} \lambda_{-}^{n}+c_{3}} \rightarrow \frac{1}{c_{3}} \text { as } n \rightarrow \infty .
$$

(b) If $b>\frac{3}{2}$, then we have $0<\left|\lambda_{0}\right|<\lambda-=1<\frac{2 b}{3}<\lambda_{+}$, from which the result follows.

3. If $a<-1+b$, then $\lambda_{-}<1<\lambda_{+}$. That is $\lambda_{+}^{n} \rightarrow \infty$ and the result follows.

In the following results, we show that when $a>\frac{4}{27} b^{3}$, under certain conditions there exist solutions, either periodic or converge to periodic solutions for equation (1).

Suppose that $\varphi=\frac{p}{q} \pi$, where $p$ and $q$ are positive relatively prime integers such that $0<p<\frac{q}{2}$.

Theorem 7. Assume that $a>\frac{4}{27} b^{3}, a<b+1$. Let $\left\{x_{n}\right\}_{n=-2}^{\infty}$ be a solution of equation (1) such that $\left(x_{0}, x_{-1}, x_{-2}\right) \notin D \cup F$. If $a^{2}+b a-1=0$, then $\left\{x_{n}\right\}_{n=-2}^{\infty}$ converges to a periodic solution with prime period $2 q$. 
Proof. Assume that $\left\{x_{n}\right\}_{n=-2}^{\infty}$ is a solution of equation (1) such that $\left(x_{0}, x_{-1}, x_{-2}\right) \notin$ $D \cup F$ and let the angle $\left.\varphi=\frac{p}{q} \pi \in\right] 0, \frac{\pi}{2}[$.

When $a>\frac{4}{27} b^{3}$ and $a^{2}+b a-1=0\left(\lambda_{0}=-a>-1\right)$, the solution of equation (1) is

$$
x_{n}=\frac{1}{c_{1} \lambda_{0}^{n}+c_{2} \cos n \varphi+c_{3} \sin n \varphi} .
$$

Then we can write

$$
\begin{aligned}
x_{2 q m+l} & =\frac{1}{c_{1} \lambda_{0}^{2 q m+l}+c_{2} \cos (2 q m+l) \varphi+c_{3} \sin (2 q m+l) \varphi} \\
& =\frac{1}{c_{1} \lambda_{0}^{2 q m+l}+c_{2} \cos l \varphi+c_{3} \sin l \varphi}, l=1,2, \ldots, 2 q .
\end{aligned}
$$

As $m \rightarrow \infty$, we get

$$
x_{2 q m+l} \rightarrow \mu_{l}=\frac{1}{c_{2} \cos l \varphi+c_{3} \sin l \varphi}, l=1,2, \ldots, 2 q .
$$

Therefore, the solution $\left\{x_{n}\right\}_{n=-2}^{\infty}$ converges to

$$
\left\{\ldots, \mu_{1}, \mu_{2}, \ldots, \mu_{2 q-1}, \mu_{2 q}, \mu_{1}, \mu_{2}, \ldots, \mu_{2 q-1}, \mu_{2 q}, \ldots\right\} .
$$

Simple calculations show that the solution $(21)$ is a period- $2 q$ solution for equation (1) and will be omitted.

This completes the proof.

Theorem 8. Assume that $a>\frac{4}{27} b^{3}, a<b+1$ and $a^{2}+b a-1=0$. Let $\left\{x_{n}\right\}_{n=-2}^{\infty}$ be a solution of equation (1) such that $\left(x_{0}, x_{-1}, x_{-2}\right) \notin F$. If $\left(x_{0}, x_{-1}, x_{-2}\right) \in D$, then $\left\{x_{n}\right\}_{n=-2}^{\infty}$ is a periodic solution with prime period $2 q$.

Proof. Assume that $\left\{x_{n}\right\}_{n=-2}^{\infty}$ is a solution of equation (1) such that $\left(x_{0}, x_{-1}, x_{-2}\right) \notin$ $F$ and let the angle $\left.\varphi=\frac{p}{q} \pi \in\right] 0, \frac{\pi}{2}[$.

When $\left(x_{0}, x_{-1}, x_{-2}\right) \in D$, we have that $c_{1}=0$ and the solution of equation (1) is

$$
x_{n}=\frac{1}{c_{2} \cos n \varphi+c_{3} \sin n \varphi} .
$$

Then we have

$$
\begin{aligned}
x_{n+2 q} & =\frac{1}{c_{2} \cos (n+2 q) \varphi+c_{3} \sin (n+2 q) \varphi} \\
& =\frac{1}{c_{2} \cos (n \varphi+2 p \pi)+c_{3} \sin (n \varphi+2 p \pi)} \\
& =\frac{1}{c_{2} \cos (n \varphi)+c_{3} \sin (n \varphi)} \\
& =x_{n} .
\end{aligned}
$$


This completes the proof.

Example (1) Figure 1. shows that if $a=b=\frac{1}{\sqrt{2}},\left(a>\frac{4}{27} b^{3}, a<b+1, a^{2}+a b-1=0\right.$ and $\varphi=\frac{1}{4} \pi$ ), then a solution $\left\{x_{n}\right\}_{n=-2}^{\infty}$ of equation (1) with initial conditions $x_{-2}=2, x_{-1}=0.1$ and $x_{0}=1$ converges to a period- 8 solution.

Example (2) Figure 2. shows that if $a=\frac{1}{\sqrt{3}}, b=\frac{2}{\sqrt{3}}\left(a>\frac{4}{27} b^{3}, a<b+1\right.$, $a^{2}+a b-1=0$ and $\left.\varphi=\frac{1}{6} \pi\right)$, then a solution $\left\{x_{n}\right\}_{n=-2}^{\infty}$ of equation (1) with initial conditions $x_{-2}=-\frac{1}{3}, x_{-1}=-\frac{\sqrt{3}}{2}$ and $x_{0}=1\left(\left(x_{-2}, x_{-1}, x_{0}\right) \in D\right)$ is periodic with prime period-12 solution.

Example (3) Figure 3. shows that if $a=b=1,\left(a>\frac{4}{27} b^{3}, a<b+1, a^{2}+a b-1>0\right.$, then a solution $\left\{x_{n}\right\}_{n=-2}^{\infty}$ of equation (1) with initial conditions $x_{-2}=-0.2, x_{-1}=$ 2.1 and $x_{0}=2.82$ converges to zero.

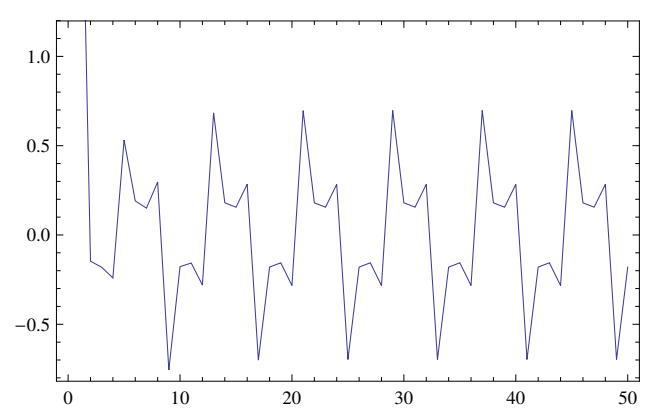

Figure 1: $\quad x_{n+1}=\frac{x_{n} x_{n-2}}{-\frac{1}{\sqrt{2}} x_{n}+\frac{1}{\sqrt{2}} x_{n-2}}$

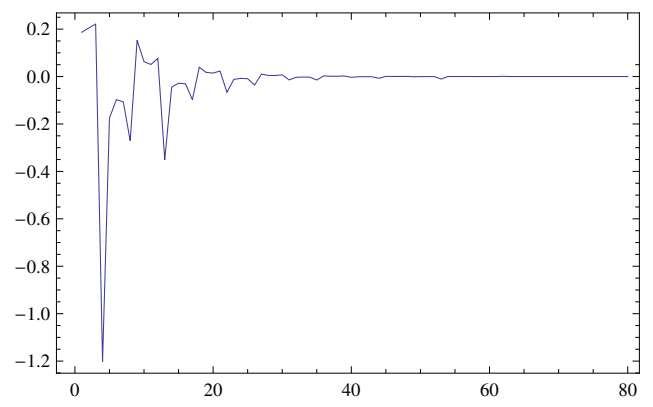

Figure 3: $\quad x_{n+1}=\frac{x_{n} x_{n-2}}{-x_{n}+x_{n-2}}$

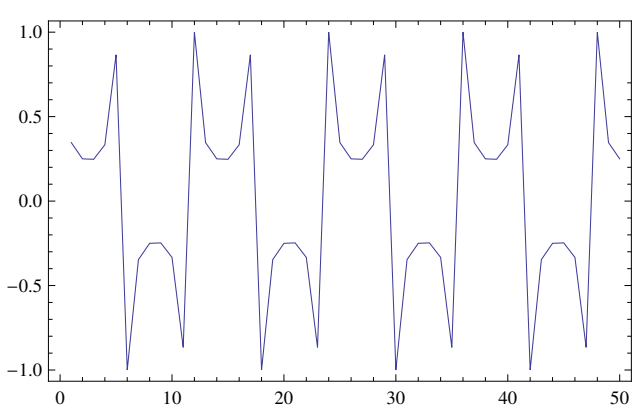

Figure 2: $\quad x_{n+1}=\frac{x_{n} x_{n-2}}{-\frac{1}{\sqrt{3}} x_{n}+\frac{2}{\sqrt{3}} x_{n-2}}$ 
R. Abo-Zeid - On a third order difference equation

\section{REFERENCES}

[1] R. Abo-Zeid, Global behavior of a higher order rational difference equation, Filomat 30 (12) (2016), 3265 - 3276.

[2] R. Abo-Zeid, Global behavior of a third order rational difference equation, Math. Bohemica, 139 (1) (2014), 25 - 37.

[3] R. Abo-Zeid, Global behavior of a rational difference equation with quadratic term, Math. Moravica, 18 (1) (2014), $81-88$.

[4] R. Abo-Zeid, On the solutions of two third order recursive sequences, Armenian J. Math., 6 (2) (2014), $64-66$.

[5] R. Abo-Zeid, Global behavior of a fourth order difference equation, Acta Commentaiones Univ. Tartuensis Math., 18 (2) (2014), 211 - 220.

[6] R. Abo-Zeid, Global Behavior of a fourth order Difference Equation with Quadratic Term, Bol. Soc. Mat. Mexicana, DOI: 10.1007/s40590 - 017 - 0180 - 8.

[7] A.M. Amleh, E. Camouzis and G. Ladas, On the dynamics of a rational difference equations Part I, Int. J. difference Equ. 3 (2008), 1 - 35.

[8] A.M. Amleh, E. Camouzis and G. Ladas, On the dynamics of a rational difference equations Part II, Int. J. difference Equ. 3 (2008), 195 - 225.

[9] A. Anisimova and I. Bula, Some problems of second-order rational difference equations with quadratic terms, Inter. J. difference Equ.,9 (1) 2014, $11-21$.

[10] I. Bajo, Forbidden sets of planar rational systems of difference equations with common denominator, Appl. Anal. Discrete Math. 8 (2014), 16 - 32.

[11] I. Bajo and E. Liz, Global behaviour of a second-order nonlinear difference equation, J. Difference Equ. Appl., 17 (10) (2011), 1471 - 1486.

[12] I. Bajo, D. Franco and J. Perán, Dynamics of a rational system of difference equations in the plane, Adv. difference Equ., 2011, Article ID 958602, 17 pages.

[13] F. Balibrea and A. Cascales, On forbidden sets, J. difference Equ. Appl., 21, (10) (2015), $974-996$.

[14] E. Camouzis and G. Ladas, Dynamics of Third Order Rational Difference Equations: With Open Problems and Conjectures, Chapman \& Hall/CRC, Boca Raton, FL, 2008.

[15] M. Dehghan, C.M. Kent, R. Mazrooei-Sebdani, N.L. Ortiz and H. Sedaghat, Dynamics of rational difference equations containing quadratic terms, J. difference Equ. Appl., 14 (2) (2008), 191 - 208.

[16] E.A. Jankowski and M.R.S. Kulenović, Attractivity and global stability for linearizable difference equations, Comput. Math. Appl. 57 (2009), 1592 - 1607.

[17] C.M. Kent and H. Sedaghat, Global attractivity in a quadratic-linear rational difference equation with delay, J. difference Equ. Appl., 15, (10) (2009), 913 - 925. 
[18] V. L. Kocic, G. Ladas, Global Behavior of Nonlinear Difference Equations of Higher Order with Applications, Kluwer Academic, Dordrecht, 1993.

[19] V.L. Kocic, G. Ladas, Global attractivity in a second order nonlinear difference equation, J. Math. Anal. Appl., 180 (1993), $144-150$.

[20] M.R.S. Kulenović and G. Ladas, Dynamics of Second Order Rational Difference Equations; With Open Problems and Conjectures, Chapman and Hall/HRC Boca Raton, 2002.

[21] H. Sedaghat, On third order rational equations with quadratic terms, J. difference Equ. Appl. 14 (8) (2008), 889 - 897.

[22] H. Shojaei, S. Parvandeh, T. Mohammadi, Z. Mohammadi and N. Mohammadi, Stability and convergence of a higher order rational difference equation, Australian J. Bas. Appl. Sci., 5 (11) (2011), $72-77$.

[23] I. Szalkai, Avoiding forbidden sequences by finding suitable initial values, Inter. J. difference Equ., 3 (2) (2008), $305-315$.

\section{R. Abo-Zeid}

Department of Basic Science

The Higher Institute for Engineering \& Technology, Al-Obour

Cairo, Egypt

email: abuzead73@yahoo.com 